\title{
Early glycaemic control in metformin users receiving their first add-on therapy: a population-based study of 4,734 people with type 2 diabetes
}

\author{
Reimar W. Thomsen ${ }^{1} \cdot$ Lisbeth M. Baggesen $^{1} \cdot$ Mette Søgaard $^{1} \cdot$ Lars Pedersen $^{1}$. \\ Helene Nørrelund $^{1} \cdot$ Esben S. Buhl $^{2}$ - Christiane L. Haase ${ }^{2}$ - Søren P. Johnsen ${ }^{1}$
}

Received: 27 April 2015 / Accepted: 26 June 2015 /Published online: 16 August 2015

(C) Springer-Verlag Berlin Heidelberg 2015

\begin{abstract}
Aims/hypothesis The aims of this work were to assess glycaemic control in metformin users receiving their first add-on glucose-lowering therapy and to examine the real-life effectiveness of different add-on drugs.

Methods We carried out a population-based cohort study using healthcare databases in northern Denmark during 2000-2012. We included 4,734 persons who initiated metformin monotherapy and added another glucose-lowering drug within 3 years. Attainment of recommended $\mathrm{HbA}_{1 \mathrm{c}}$ goals within 6 months of add-on was investigated, using Poisson regression analysis adjusted for age, sex, baseline $\mathrm{HbA}_{1 \mathrm{c}}$, diabetes duration, complications and Charlson Comorbidity Index.

Results Median metformin treatment duration at intensification was 12 months (interquartile range [IQR] 4-23 months) and pre-intensification $\mathrm{HbA}_{1 \mathrm{c}}$ was $8.0 \%$ (IQR 7.2-9.2\%) (64 [IQR 55-77] mmol/mol). Median $\mathrm{HbA}_{1 \mathrm{c}}$ dropped $1.2 \%$ (13 $\mathrm{mmol} / \mathrm{mol}$ ) with a sulfonylurea (SU) add-on, $0.8 \%$ $(9 \mathrm{mmol} / \mathrm{mol})$ with a dipeptidyl peptidase-4 (DPP-4) inhibitor, $1.3 \%(14 \mathrm{mmol} / \mathrm{mol}$ ) with a glucagon-like peptide-1 (GLP-1) receptor agonist, $0.9 \%(10 \mathrm{mmol} / \mathrm{mol})$ with other non-insulin
\end{abstract}

Electronic supplementary material The online version of this article (doi:10.1007/s00125-015-3698-1) contains peer-reviewed but unedited supplementary material, which is available to authorised users.

Reimar W. Thomsen

rwt@clin.au.dk

1 Department of Clinical Epidemiology, Institute of Clinical Medicine, Aarhus University Hospital, Olof Palmes Allé 43-45, 8200 Aarhus $\mathrm{N}$, Denmark

2 Novo Nordisk Scandinavia AB, Ørestad, Copenhagen, Denmark drugs and $2.4 \%(26 \mathrm{mmol} / \mathrm{mol})$ with insulin. Compared with SU add-on, attainment of $\mathrm{HbA}_{1 \mathrm{c}}<7 \%(<53 \mathrm{mmol} / \mathrm{mol})$ was higher with GLP-1 receptor agonists (adjusted RR [aRR] $1.10 ; 95 \%$ CI 1.01, 1.19) and lower with DPP-4 inhibitors (aRR 0.94; 95\% CI 0.89, 0.99), other drugs (aRR 0.86; 95\% CI $0.77,0.96$ ) and insulin (aRR $0.88 ; 95 \%$ CI $0.77,0.99$ ). The proportion of metformin add-on users who attained $\mathrm{HbA}_{1 \mathrm{c}}$ $<7 \%$ ( $<53 \mathrm{mmol} / \mathrm{mol}$ ) increased from $46 \%$ in $2000-2003$ to $59 \%$ in $2010-2012$, whereas attainment of $\mathrm{HbA}_{1 \mathrm{c}}<6.5 \%$ $(<48 \mathrm{mmol} / \mathrm{mol})$ remained $30 \%$ among patients aged $<65$ years without comorbidities.

Conclusions/interpretation Among early type 2 diabetes patients receiving their first metformin add-on treatment, $\mathrm{HbA}_{1 \mathrm{c}}$ reduction with different non-insulin drugs is similar to, and comparable with, that observed in randomised trials, yet $41 \%$ do not achieve $\mathrm{HbA}_{1 \mathrm{c}}<7 \%(<53 \mathrm{mmol} / \mathrm{mol})$ within 6 months.

Keywords Antidiabetic agents · Clinical quality · Comparative effectiveness - Glucose-lowering therapy · Glycaemic control $\cdot \mathrm{HbA}_{1 \mathrm{c}} \cdot$ Hypoglycaemic agents . Metformin · Pharmacoepidemiology · Type 2 diabetes
Abbreviations
aRR Adjusted relative risk
CCI Charlson Comorbidity Index
DPP-4 Dipeptidyl peptidase-4
eGFR Estimated GFR
GLD Glucose-lowering drug
GLP-1 Glucagon-like peptide-1
IQR Interquartile range
SU Sulfonylurea 


\section{Introduction}

Metformin monotherapy is currently the recommended initial glucose-lowering drug (GLD) for most patients with type 2 diabetes, with addition of a second GLD if $\mathrm{HbA}_{1 \mathrm{c}}$ targets are not achieved. Glucose-lowering treatment options have expanded considerably [1] yet the number of head-to-head trials comparing GLDs is limited and there is little evidence to guide clinicians' choice of add-on therapy [2]. Adding a non-insulin second-line GLD to metformin was found to reduce $\mathrm{HbA}_{1 \mathrm{c}}$ levels by approximately $1 \%$ in clinical trials [3] and an individualised treatment approach following metformin initiation is currently recommended [4]. In real-world type 2 diabetes populations, who may differ from clinical trial participants in some characteristics (e.g. age, comorbidities and compliance with medications [5]), little is known about success rates when older and newer GLDs are used to intensify metformin therapy.

We aimed to assess the quality of glycaemic control in a large population-based cohort of incident metformin users receiving their first add-on glucose-lowering therapy. We examined the association of calendar time with achieved glycaemic control and compared the reduction of $\mathrm{HbA}_{1 \mathrm{c}}$ for different add-on GLDs.

\section{Methods}

Setting and study population We conducted this cohort study in northern Denmark during 2000-2012. We used linkage of existing population-based medical databases covering 1.8 million inhabitants or $30 \%$ of Denmark's population, as described in more detail elsewhere [6]. The cohort included individuals who redeemed a first-time GLD prescription (codes A10A, A10B) of metformin monotherapy (no GLD prescription other than metformin within the first 14 days) between 1 January 2000 and 31 December 2012 and who had at least 12 months of baseline data. Metformin exposure time was estimated as the quantity filled at each prescription collection, divided by the minimum daily dose used in clinical practice $(500 \mathrm{mg}$ ), plus another 100 days to avoid falsely assumed termination of treatment due to non-compliance [7].

The treatment-intensification cohort consisted of metformin-exposed patients, who added another GLD within the first 3 years and had at least one $\mathrm{HbA}_{1 \mathrm{c}}$ value recorded within 12 months before and within 3-6 months after the addon prescription date in a regional laboratory information system research database [6] (for patient flow, see electronic supplementary material [ESM] Fig. 1).

Add-on drugs Metformin add-on therapies examined were sulfonylurea (SU), dipeptidyl peptidase-4 (DPP-4) inhibitors, glucagon-like peptide-1 (GLP-1) receptor agonists, other non- insulin GLDs and insulin. The first intensification drug defined add-on exposure and subsequent changes were ignored, an approach similar to intention-to-treat analyses.

$\mathbf{H b A}_{1 \mathrm{c}}$ outcomes Baseline $\mathrm{HbA}_{1 \mathrm{c}}$ was defined as the most recently measured $\mathrm{HbA}_{1 \mathrm{c}}$ value within 12 months prior to the intensification date. We assessed the $\mathrm{HbA}_{1 \mathrm{c}}$ level achieved between 2 and 6 months (60-180 days) following the intensification date. If there were several $\mathrm{HbA}_{1 \mathrm{c}}$ measurements, the value closest to day 180 was used. $\mathrm{HbA}_{1 \mathrm{c}}$ was analysed by either the HPLC method or immunoassays on automated equipment.

Patient characteristics We obtained data from medical databases on 19 major disease categories included in the Charlson Comorbidity Index (CCI) based on patients' entire hospital contact history within 5 years before the intensification date. We separately ascertained contacts and/or prescription history for mental disorders and alcoholism-related disorders (see ESM Table 1 for codes), and for any macrovascular or microvascular diabetes complication [6] including prior clinical biochemical indication of renal disease ( 2 days with estimated glomerular filtration rate $[\mathrm{eGFR}] \leq 60 \mathrm{ml} \mathrm{\textrm {min } ^ { - 1 }}$ $[1.73 \mathrm{~m}]^{-2}$ and/or 2 days with urinary albumin above the cut-off for microalbuminuria). The duration of healthcaresystem-recorded diabetes was the time between the first-ever GLD prescription/hospital diagnosis of diabetes and the therapy intensification date.

Statistical analysis For each metformin add-on therapy group, we computed absolute reduction in median $\mathrm{HbA}_{1 \mathrm{c}}$ from baseline, as well as proportions of patients and RRs with $9 \%$ CIs of achieving $\mathrm{HbA}_{1 \mathrm{c}}<6.5 \%(<48 \mathrm{mmol} / \mathrm{mol})$ and $<7.0 \%(<53 \mathrm{mmol} / \mathrm{mol})$ with SU add-on as reference category. RRs were adjusted by Poisson regression for predefined factors (age [ $\leq 60$ years, $61-70$ years, $>70$ years], sex, diabetes duration $[\leq 1$ year, $>1$ year], macrovascular complications [yes/no], microvascular complications [yes/no], CCI level $[0, \geq 1]$ and baseline $\left.\mathrm{HbA}_{1 \mathrm{c}}[\leq 7.5 \%, 7.6-9.0 \%,>9.0 \%]\right)$. Analyses of $\mathrm{HbA}_{1 \mathrm{c}}<6.5 \%(<48 \mathrm{mmol} / \mathrm{mol})$ were restricted to a subgroup of 'healthy' intensification patients (i.e. aged $<65$ years, $\mathrm{CCI}=0$, no diabetes complications, no mental or alcoholism-related disorders), for whom providers might reasonably suggest a stringent $\mathrm{HbA}_{1 \mathrm{c}}$ goal [4]. Pre-intensification $\mathrm{HbA}_{1 \mathrm{c}}$ values and proportions attaining $\mathrm{HbA}_{1 \mathrm{c}}<7 \%$ $(<53 \mathrm{mmol} / \mathrm{mol})$ and, among 'healthy' patients, $<6.5 \%$ ( $<48 \mathrm{mmol} / \mathrm{mol}$ ), were assessed by calendar year periods. The study was approved by the Danish Data Protection Agency (J. no. 2013-41-1924) and needed no further ethics approval according to Danish law. 
Table 1 Characteristics of 4,734 incident metformin users at the time of treatment intensification

Variable

Add-on medication to metformin

\begin{tabular}{llllll}
\hline $\begin{array}{l}\text { Sulfonylurea } \\
(n=2,484)\end{array}$ & $\begin{array}{l}\text { DPP-4 } \\
\text { inhibitor } \\
(n=1,262)\end{array}$ & $\begin{array}{l}\text { GLP-1 RA } \\
(n=329)\end{array}$ & $\begin{array}{l}\text { Insulin } \\
(n=377)\end{array}$ & $\begin{array}{l}\text { Other GLD } \\
\text { add-on } \\
(n=282)\end{array}$ & Total $(N=4,734)$ \\
& & &
\end{tabular}

Sex

\section{Female \\ Male}

Median age at add-on, years

Age group

$$
\begin{aligned}
& <30 \text { years } \\
& \geq 30-59 \text { years } \\
& \geq 60-70 \text { years } \\
& >70 \text { years }
\end{aligned}
$$

Year of add-on commencement 2000-2003

2004-2006

2007-2009

2010-2012

Add-on baseline $\mathrm{HbA}_{1 \mathrm{c}}, \%$

Add-on baseline $\mathrm{HbA}_{1 \mathrm{c}}, \mathrm{mmol} / \mathrm{mol}$

Add-on baseline $\mathrm{HbA}_{1 \mathrm{c}}$ group

$<6.5 \%(<48 \mathrm{mmol} / \mathrm{mol})$

$6.5-6.9 \%(48-52 \mathrm{mmol} / \mathrm{mol})$

$7-7.4 \%(53-57 \mathrm{mmol} / \mathrm{mol})$

$7.5-7.9 \%(59-63 \mathrm{mmol} / \mathrm{mol})$

$8.0-8.9 \%(64-74 \mathrm{mmol} / \mathrm{mol})$

$9.0-9.9 \%(75-85 \mathrm{mmol} / \mathrm{mol})$

$\geq 10.0 \%(\geq 86 \mathrm{mmol} / \mathrm{mol})$

Diabetes duration

$$
\begin{aligned}
& <1 \text { year } \\
& 1-2 \text { years } \\
& 2-3 \text { years } \\
& \geq 3 \text { years }
\end{aligned}
$$

Hospital outpatient clinic diagnosis of diabetes $\leq 1$ year before add-on Followed in hospital outpatient clinic in the year after add-on

Any macrovascular diabetes complication

Any microvascular diabetes complication

Neurological complications

Eye complications

Renal complications

Microalbuminuria

eGFR $\leq 60 \mathrm{ml} \mathrm{min}^{-1}(1.73 \mathrm{~m})^{-2}$

CCI score (excluding diabetes)

$$
0
$$

\begin{tabular}{|c|c|c|c|c|c|}
\hline 983 (39.6) & $460(36.5)$ & 118 (35.9) & $154(40.8)$ & $139(49.3)$ & $1,854(39.2)$ \\
\hline $1,501(60.4)$ & $802(63.5)$ & $211(64.1)$ & $223(59.2)$ & $143(50.7)$ & $2,880(60.8)$ \\
\hline 59.0 & 59.6 & 54.2 & 54.4 & 58.9 & 58.6 \\
\hline $16(0.6)$ & $15(1.2)$ & $6(1.8)$ & $13(3.4)$ & $3(1.1)$ & $53(1.1)$ \\
\hline $1,310(52.7)$ & $632(50.1)$ & $209(63.5)$ & $220(58.4)$ & $148(52.5)$ & $2,519(53.2)$ \\
\hline 742 (29.9) & $414(32.8)$ & $99(30.1)$ & $84(22.3)$ & $86(30.5)$ & $1,425(30.1)$ \\
\hline $416(16.7)$ & $201(15.9)$ & 15 (4.6) & $60(15.9)$ & $45(16.0)$ & 737 (15.6) \\
\hline $293(11.8)$ & $0(0)$ & $0(0)$ & $20(5.3)$ & $23(8.2)$ & $336(7.1)$ \\
\hline $604(24.3)$ & $0(0)$ & $0(0)$ & $77(20.4)$ & $105(37.2)$ & 786 (16.6) \\
\hline $919(37.0)$ & $455(36.1)$ & $42(12.8)$ & $137(36.3)$ & $132(46.8)$ & $1,685(35.6)$ \\
\hline 668 (26.9) & 807 (63.9) & 287 (87.2) & 143 (37.9) & $22(7.8)$ & $1,927(40.7)$ \\
\hline $8.0(7.3-9.2)$ & $7.6(7.0-8.6)$ & $8.0(7.2-9.2)$ & $9.6(7.9-11.0)$ & $7.9(7.2-9.1)$ & $8.0(7.2-9.2)$ \\
\hline $64(56-77)$ & $60(53-70)$ & $64(55-77)$ & 81 (63-97) & $63(55-76)$ & $64(55-77)$ \\
\hline $99(4.0)$ & $69(5.5)$ & $26(7.9)$ & $10(2.7)$ & $20(7.1)$ & $224(4.7)$ \\
\hline $244(9.8)$ & 184 (14.6) & $27(8.2)$ & $21(5.6)$ & $28(9.9)$ & 504 (10.6) \\
\hline $446(18.0)$ & $269(21.3)$ & 55 (16.7) & $26(6.9)$ & $53(18.8)$ & 849 (17.9) \\
\hline 408 (16.4) & $239(18.9)$ & $55(16.7)$ & $38(10.1)$ & $42(14.9)$ & $782(16.5)$ \\
\hline $548(22.1)$ & $261(20.7)$ & $75(22.8)$ & $56(14.9)$ & $60(21.3)$ & $1,000(21.1)$ \\
\hline $342(13.8)$ & $130(10.3)$ & $37(11.2)$ & $67(17.8)$ & $37(13.1)$ & 613 (12.9) \\
\hline $397(16.0)$ & $110(8.7)$ & 54 (16.4) & $159(42.2)$ & $42(14.9)$ & $762(16.1)$ \\
\hline $1,152(46.4)$ & $536(42.5)$ & $118(35.9)$ & $185(49.1)$ & $101(35.8)$ & $2,092(44.2)$ \\
\hline $636(25.6)$ & 333 (26.4) & $107(32.5)$ & $72(19.1)$ & $84(29.8)$ & $1,232(26.0)$ \\
\hline 445 (17.9) & $280(22.2)$ & $71(21.6)$ & $79(21.0)$ & $60(21.3)$ & 935 (19.8) \\
\hline $251(10.1)$ & $113(9.0)$ & $33(10.0)$ & $41(10.9)$ & $37(13.1)$ & $475(10.0)$ \\
\hline $430(17.3)$ & $168(13.3)$ & 104 (31.6) & 143 (37.9) & 33 (11.7) & $878(18.5)$ \\
\hline $416(16.7)$ & $143(11.3)$ & $60(18.2)$ & 104 (27.6) & $54(19.1)$ & 777 (16.4) \\
\hline $361(14.5)$ & 169 (13.4) & $56(17.0)$ & 75 (19.9) & $45(16.0)$ & 706 (14.9) \\
\hline $700(28.2)$ & $317(25.1)$ & $92(28.0)$ & $148(39.3)$ & $73(25.9)$ & $1,330(28.1)$ \\
\hline $29(1.2)$ & $4(0.3)$ & $2(0.6)$ & $9(2.4)$ & $0(0)$ & $44(0.9)$ \\
\hline $261(10.5)$ & $103(8.2)$ & $31(9.4)$ & $69(18.3)$ & $28(9.9)$ & $492(10.4)$ \\
\hline $24(1.0)$ & $8(0.6)$ & $3(0.9)$ & $11(2.9)$ & $3(1.1)$ & $49(1.0)$ \\
\hline $317(12.8)$ & $164(13.0)$ & $63(19.1)$ & $58(15.4)$ & $33(11.7)$ & 635 (13.4) \\
\hline $222(8.9)$ & $89(7.1)$ & $10(3.0)$ & $41(10.9)$ & $23(8.2)$ & $385(8.1)$ \\
\hline 1,977 (79.6) & $1,036(82.1)$ & $268(81.5)$ & $276(73.2)$ & $227(80.5)$ & $3,784(79.9)$ \\
\hline 333 (13.4) & $141(11.2)$ & $43(13.1)$ & $51(13.5)$ & $37(13.1)$ & $605(12.8)$ \\
\hline $130(5.2)$ & $65(5.2)$ & $8(2.4)$ & $22(5.8)$ & $13(4.6)$ & $238(5.0)$ \\
\hline $44(1.8)$ & $20(1.6)$ & $10(3.0)$ & $28(7.4)$ & $5(1.8)$ & $107(2.3)$ \\
\hline $61(2.5)$ & $31(2.5)$ & $8(2.4)$ & $15(4.0)$ & $13(4.6)$ & $128(2.7)$ \\
\hline $105(4.2)$ & $32(2.5)$ & $13(4.0)$ & $17(4.5)$ & $10(3.5)$ & $177(3.7)$ \\
\hline $56(2.3)$ & $25(2.0)$ & $7(2.1)$ & $14(3.7)$ & $2.1(0.7)$ & $104(2.2)$ \\
\hline 73 (2.9) & $30(2.4)$ & $5(1.5)$ & $16(4.2)$ & $3(1.1)$ & $127(2.7)$ \\
\hline
\end{tabular}

1

2

$$
\geq 3
$$

Previous myocardial infarction

Cerebrovascular disease

Peripheral vascular disease

Chronic heart failure 
Table 1 (continued)

\begin{tabular}{|c|c|c|c|c|c|c|}
\hline \multirow[t]{2}{*}{ Variable } & \multicolumn{6}{|c|}{ Add-on medication to metformin } \\
\hline & $\begin{array}{l}\text { Sulfonylurea } \\
(n=2,484)\end{array}$ & $\begin{array}{l}\text { DPP-4 } \\
\text { inhibitor } \\
(n=1,262)\end{array}$ & $\begin{array}{l}\text { GLP-1 RA } \\
(n=329)\end{array}$ & $\begin{array}{l}\text { Insulin } \\
(n=377)\end{array}$ & $\begin{array}{l}\text { Other GLD } \\
\text { add-on } \\
(n=282)\end{array}$ & Total $(N=4,734)$ \\
\hline Atrial fibrillation & $123(5.0)$ & $61(4.8)$ & $12(3.6)$ & $17(4.5)$ & $14(5.0)$ & $227(4.8)$ \\
\hline Hypertension & $438(17.6)$ & $226(17.9)$ & $74(22.5)$ & $90(23.9)$ & $48(17.0)$ & $876(18.5)$ \\
\hline Chronic obstructive pulmonary disease & $128(5.2)$ & $60(4.8)$ & $13(4.0)$ & $37(9.8)$ & $13(4.6)$ & $251(5.3)$ \\
\hline Cancer & $79(3.2)$ & $49(3.9)$ & $11(3.3)$ & $23(6.1)$ & $8(2.8)$ & $170(3.6)$ \\
\hline Renal disease & $15(0.6)$ & $4(0.3)$ & $2(0.6)$ & $7(1.9)$ & $4(1.4)$ & $32(0.7)$ \\
\hline Rheumatic disease & $43(1.7)$ & $16(1.3)$ & $4(1.2)$ & $13(3.4)$ & $5(1.8)$ & $81(1.7)$ \\
\hline Osteoarthritis & $179(7.2)$ & $97(7.7)$ & $30(9.1)$ & $35(9.3)$ & $27(9.6)$ & $368(7.8)$ \\
\hline Osteoporosis/fracture & $28(1.1)$ & $16(1.3)$ & $5(1.5)$ & $7(1.9)$ & $2(0.7)$ & $58(1.2)$ \\
\hline History of infections requiring hospitalisation & $380(15.3)$ & $167(13.2)$ & $55(16.7)$ & $75(19.9)$ & $39(13.8)$ & $716(15.1)$ \\
\hline Obesity & $253(10.2)$ & $98(7.8)$ & $65(19.8)$ & $67(17.8)$ & $40(14.2)$ & $523(11.0)$ \\
\hline Alcoholism & $20(0.8)$ & $6(0.5)$ & $3(0.9)$ & $6(1.6)$ & $0(0)$ & $35(0.7)$ \\
\hline Mental disorders & $608(24.5)$ & $344(27.3)$ & $96(29.2)$ & $114(30.2)$ & $78(27.7)$ & $1,240(26.2)$ \\
\hline Previous hypoglycaemia & $2(0.1)$ & $2(0.2)$ & $0(0)$ & $0(0)$ & $1(0.4)$ & $5(0.1)$ \\
\hline Any macroangiopathy & $380(15.3)$ & $175(13.9)$ & $56(17.0)$ & $80(21.2)$ & $46(16.3)$ & $737(15.6)$ \\
\hline Thrombocyte aggregation prophylaxis & $946(38.1)$ & $499(39.5)$ & $130(39.5)$ & $146(38.7)$ & $111(39.4)$ & $1,832(38.7)$ \\
\hline Statin & $1,593(64.1)$ & $947(75.0)$ & $257(78.1)$ & $242(64.2)$ & $196(69.5)$ & $3,235(68.3)$ \\
\hline Any antihypertensive treatment & $1,772(71.3)$ & $948(75.1)$ & $245(74.5)$ & $252(66.8)$ & $215(76.2)$ & $3,432(72.5)$ \\
\hline ACE inhibitor & $1,025(41.3)$ & $538(42.6)$ & $149(45.3)$ & $147(39.0)$ & $119(42.2)$ & $1,978(41.8)$ \\
\hline ATII antagonist & $559(22.5)$ & $355(28.1)$ & $81(24.6)$ & $82(21.8)$ & $73(25.9)$ & $1,150(24.3)$ \\
\hline Oral steroids & $193(7.8)$ & $92(7.3)$ & $26(7.9)$ & $59(15.6)$ & $18(6.4)$ & $388(8.2)$ \\
\hline \multicolumn{7}{|l|}{ Marital status } \\
\hline Unmarried & $432(17.4)$ & $227(18.0)$ & $83(25.2)$ & $92(24.4)$ & $42(14.9)$ & $876(18.5)$ \\
\hline Widowed & $244(9.8)$ & $110(8.7)$ & $20(6.1)$ & $32(8.5)$ & $31(11.0)$ & $437(9.2)$ \\
\hline Divorced & $332(13.4)$ & $176(13.9)$ & $46(14.0)$ & $56(14.9)$ & $49(17.4)$ & $659(13.9)$ \\
\hline Married & $1,476(59.4)$ & $749(59.4)$ & $180(54.7)$ & $197(52.3)$ & $160(56.7)$ & $2,762(58.3)$ \\
\hline
\end{tabular}

Data are shown as $n(\%)$ or as median (IQR)

${ }^{a}$ The CCI includes 19 major disease categories, ascertained from each individual's complete hospital contact history within 5 years before the date of add-on glucose-lowering treatment. Diabetes was excluded, see text

ACE, angiotensin-converting enzyme; ATII, angiotensin II; GLP-1 RA, GLP-1 receptor antagonist

\section{Results}

Patient characteristics Among 4,734 metformin monotherapy initiators with add-on therapy and available $\mathrm{HbA}_{1 \mathrm{c}}$ measurements (ESM Fig. 1), 52\% (2,484) added an SU, 27\% $(1,262)$ a DPP-4 inhibitor, 7\% (329) a GLP-1 receptor agonist (298 liraglutide, 31 exenatide), 6\% (282) another non-insulin GLD and $8 \%$ (377) insulin. At the time of therapy intensification, the median age was 59 years (IQR 50-66 years), 20\% had CCI comorbidities other than diabetes, duration of metformin use was 12 months (IQR 4-23 months) and median baseline $\mathrm{HbA}_{1 \mathrm{c}}$ prior to add-on therapy was $8.0 \%$ (IQR 7.2-9.2\%) (64 [IQR 55-77] mmol/mol) (Table 1).

Among patients who received SU add-on, median age was 59 years, median $\mathrm{HbA}_{1 \mathrm{c}}$ was $8.0 \%$ (64 mmol/mol) and $20 \%$ had CCI comorbidities (Table 1). Corresponding figures appeared similar for patients who were intensified with
DPP-4 inhibitors (60 years, $7.6 \%$ [60 mmol $/ \mathrm{mol}]$ and $18 \%$ ), GLP-1 receptor agonists, although younger (54 years, $8.0 \%$ [64 mmol/mol] and 19) and other GLDs (59 years, 7.9\% [63 mmol $/ \mathrm{mol}$ ] and 20\%). Patients who received insulin add-on were younger, had higher baseline $\mathrm{HbA}_{1 \mathrm{c}}$ and more comorbidity (54 years, $9.6(81 \mathrm{mmol} / \mathrm{mol})$, and $27 \%)$.

$\mathrm{HbA}_{1 \mathrm{c}}$ reduction with add-on therapy Overall, median $\mathrm{HbA}_{1 \mathrm{c}}$ dropped from $8.0 \%(64 \mathrm{mmol} / \mathrm{mol})$ at baseline to $6.8 \%$ (51 mmol $/ \mathrm{mol}$ ) within 2-6 months after commencement of addon therapy, corresponding to a reduction of $1.2 \%$ (13 $\mathrm{mmol} / \mathrm{mol})$. Absolute reductions were $1.2 \%$ (13 $\mathrm{mmol} / \mathrm{mol}$ ) (from 8.0 to $6.8 \%$ [64 to $51 \mathrm{mmol} / \mathrm{mol}$ ]) for SU add-on, $0.8 \%$ (9 mmol $/ \mathrm{mol}$ ) (from 7.6 to $6.8 \%$ [60 to $51 \mathrm{mmol} / \mathrm{mol}])$ for DPP-4 inhibitors, $1.3 \%(14 \mathrm{mmol} / \mathrm{mol})$ (from 8.0 to $6.7 \%$ [64 to $50 \mathrm{mmol} / \mathrm{mol}$ ]) for GLP-1 receptor agonists including $1.3 \%$ (14 mmol $/ \mathrm{mol}$ ) (from 7.9 to $6.6 \%$ [63 


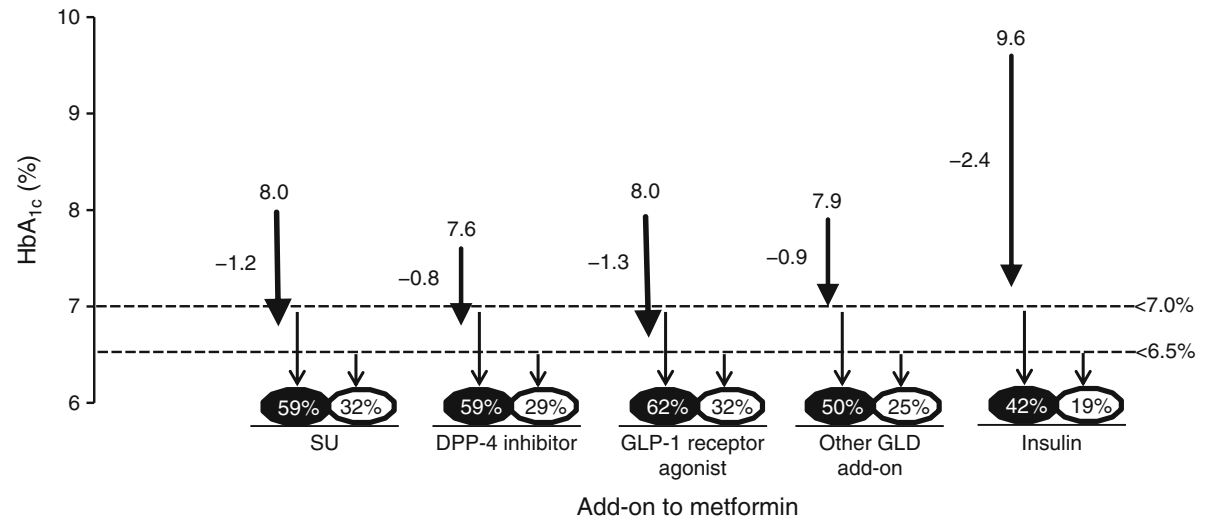

Fig. 1 Absolute reduction in median $\mathrm{HbA}_{1 \mathrm{c}}$ observed before and after add-on therapy. Large arrows show baseline $\mathrm{HbA}_{1 \mathrm{c}}(\%)$ and absolute reduction in $\mathrm{HbA}_{1 \mathrm{c}}(\%)$ from baseline to 2-6 months after treatment. The figure also shows the crude proportion of all patients (\%) reaching

to $49 \mathrm{mmol} / \mathrm{mol}$ ) for liraglutide, $0.9 \%(10 \mathrm{mmol} / \mathrm{mol})$ (from 7.9 to $7.0 \%$ [63 to $53 \mathrm{mmol} / \mathrm{mol}]$ ) for other non-insulin GLDs and $2.4 \%(26 \mathrm{mmol} / \mathrm{mol}$ ) (from 9.6 to $7.2 \%$ [81 to $55 \mathrm{mmol} / \mathrm{mol}]$ ) for insulin add-on (Fig. 1 and ESM Table 2). Overall, 2,704 (57\%) of all 4,734 metformin users who received add-on therapy reached an $\mathrm{HbA}_{1 \mathrm{c}}<7 \%$ within 2-6 months, while $466(30 \%)$ of 1,558 patients $<65$ years without recorded complications or comorbidities attained an $\mathrm{HbA}_{1 \mathrm{c}}<6.5 \%$ (ESM Table 2).

Compared with SU add-on, attainment of $\mathrm{HbA}_{1 \mathrm{c}}<7 \%$ $(<53 \mathrm{mmol} / \mathrm{mol})$ was higher with GLP-1 receptor agonists (adjusted RR [aRR] 1.10; 95\% CI 1.01, 1.19), including liraglutide (aRR 1.12; 95\% CI 1.03, 1.22), and lower with DPP-4 inhibitors (aRR 0.94; 95\% CI 0.89, 0.99), other noninsulin GLDs (aRR 0.86; 95\% CI 0.77, 0.96) and insulin (aRR 0.88; 95\% CI 0.77, 0.99) (Fig. 2 and ESM Table 2). Similar differences in attainment of an $\mathrm{HbA}_{1 \mathrm{c}}$ target $<6.5 \%$ $(<48 \mathrm{mmol} / \mathrm{mol})$ appeared among type 2 diabetes patients aged $<65$ years without complications or comorbidities (Fig. 2 and ESM Table 2), as well as in analyses restricted to the 2007-2012 period ( $n=3,612$ patients) (ESM Table 3), yet due to the smaller populations these estimates were statistically imprecise.

$\mathrm{HbA}_{1 \mathrm{c}}$ over calendar time The proportion of all metformin users who attained $\mathrm{HbA}_{1 \mathrm{c}}$ targets of $<7 \%(<53 \mathrm{mmol} / \mathrm{mol})$ and $<6.5 \%$ ( $<48 \mathrm{mmol} / \mathrm{mol}$ ), respectively, after add-on therapy increased from $46 \%$ and $26 \%$ in $2000-2003$ to $59 \%$ and $29 \%$ in 2010-2012. This apparent time trend was associated with decreasing pre-intensification $\mathrm{HbA}_{1 \mathrm{c}}$ values over time (ESM Fig. 2). Corresponding proportions for metformin users aged $<65$ years without comorbidities who attained $\mathrm{HbA}_{1 \mathrm{c}}$ $<6.5 \%(<48 \mathrm{mmol} / \mathrm{mol})$ remained around $30 \%$ with a slightly decreasing tendency (ESM Fig. 3). The median $\mathrm{HbA}_{1 \mathrm{c}}$ level achieved in 2010-2012 was 6.8\% (IQR 6.4-7.4\%) (51 [IQR $46-57] \mathrm{mmol} / \mathrm{mol}$ ) in all metformin add-on users and was identical at $6.8 \%$ (IQR 6.4-7.4\%) (51 [IQR 46-57] glycaemic targets of $\mathrm{HbA}_{1 \mathrm{c}}<7.0 \%(<53 \mathrm{mmol} / \mathrm{mol})$ (black ellipses) and/ or $\mathrm{HbA}_{1 \mathrm{c}}<6.5 \%$ ( $\left.<48 \mathrm{mmol} / \mathrm{mol}\right)$ in patients $<65$ years with no comorbidities (white ellipses). To convert values for $\mathrm{HbA}_{1 \mathrm{c}}$ in \% into $\mathrm{mmol} / \mathrm{mol}$, subtract 2.15 and multiply by 10.929

$\mathrm{mmol} / \mathrm{mol}$ ) in metformin add-on users aged $<65$ years without comorbidities.

\section{Discussion}

Among real-world metformin users with short diabetes duration ( 0 to $\leq 3$ years) who receive first add-on treatment, reduction in median $\mathrm{HbA}_{1 \mathrm{c}}$ was approximately $1 \%$ and comparable
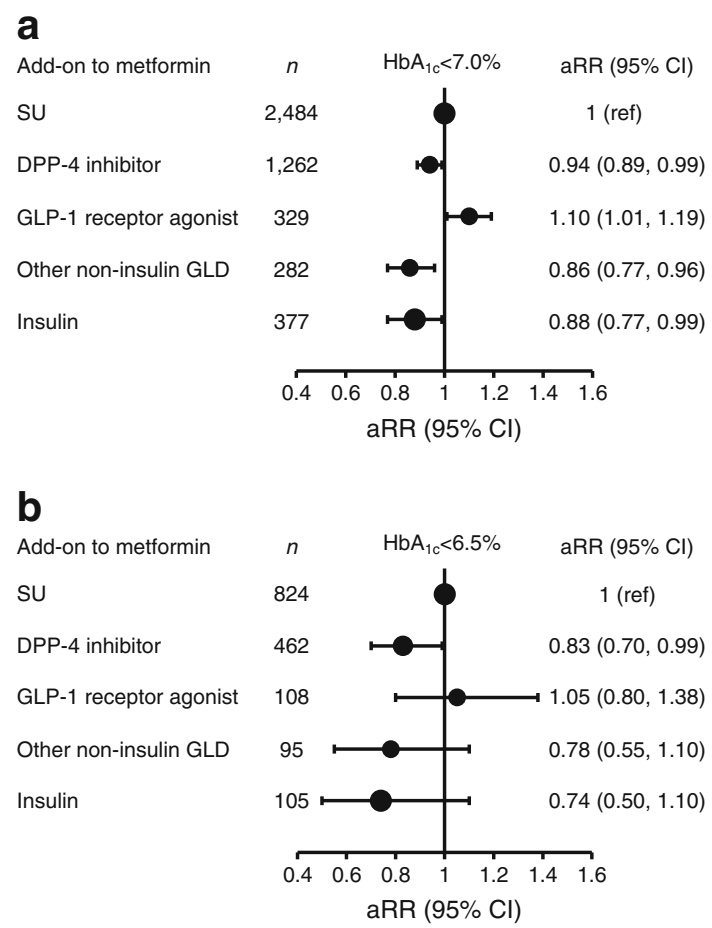

Fig. 2 aRRs for attaining $\mathrm{HbA}_{1 \mathrm{c}}$ targets of $<7 \%(<53 \mathrm{mmol} / \mathrm{mol})$ in all patients (a) and $<6.5 \%(<48 \mathrm{mmol} / \mathrm{mol})$ in patients $<65$ years with no comorbidities (b) 2-6 months after intensification. RR was adjusted for age, sex, diabetes duration, macrovascular type 2 diabetes complications, microvascular type 2 diabetes complications, CCI score and baseline $\mathrm{HbA}_{1 \mathrm{c}}$ 
with that observed in randomised clinical trials [5]. Differences between add-on treatment regimens were modest, and $\mathrm{HbA}_{1 \mathrm{c}}$ reductions with add-on of DPP-4 inhibitors $(0.8 \%$, $9 \mathrm{mmol} / \mathrm{mol}), \mathrm{SU}(1.2 \%, 13 \mathrm{mmol} / \mathrm{mol})$ and GLP-1 receptor agonists $(1.3 \%, 14 \mathrm{mmol} / \mathrm{mol})$ were similar. Nonetheless, $41 \%$ of patients in the most recent years did not achieve an $\mathrm{HbA}_{1 \mathrm{c}}<7 \%(<53 \mathrm{mmol} / \mathrm{mol})$ within 6 months.

The absolute $\mathrm{HbA}_{1 \mathrm{c}}$ reduction with insulin add-on therapy may be overestimated as it is positively correlated with baseline $\mathrm{HbA}_{1 \mathrm{c}}$ level, which was 1.5-2\% (17-22 $\left.\mathrm{mmol} / \mathrm{mol}\right)$ higher [3]. In contrast, the absolute $\mathrm{HbA}_{1 \mathrm{c}}$ reduction was similar for $\mathrm{SU}$ and GLP-1 receptor agonist add-on, although the aRR for reaching $\mathrm{HbA}_{1 \mathrm{c}}<7 \%$ ( $\left.<53 \mathrm{mmol} / \mathrm{mol}\right)$ was slightly higher with GLP-1 receptor agonists. Adjusted estimates for reaching $\mathrm{HbA}_{1 \mathrm{c}}$ targets with insulin tended to be lower, possibly related to some of the challenges that patients experience with early insulin administration in real life [8]. Nonetheless, our finding of similar $\mathrm{HbA}_{1 \mathrm{c}}$ levels achieved with insulin and other GLDs despite different $\mathrm{HbA}_{1 \mathrm{c}}$ baseline levels suggest that physicians choose effective therapy for patients with poor baseline glycaemic control, in accordance with guidelines $[4,8]$.

Few, if any, comparable population-based studies have focused on early glycaemic control in metformin initiators after intensification. Across 11 US health systems, in 2005-2009, 48\% achieved an $\mathrm{HbA}_{1 \mathrm{c}}<7 \%(<53 \mathrm{mmol} / \mathrm{mol})$ among a subgroup of 3,135 patients who initiated monotherapy and then added an oral agent within 12 months [9], which is comparable with the findings of the current study.

It is encouraging that an increasing share of patients reach $\mathrm{HbA}_{1 \mathrm{c}}<7 \%(<53 \mathrm{mmol} / \mathrm{mol})$ after therapy intensification. This may be related to decreasing pre-intensification $\mathrm{HbA}_{1 \mathrm{c}}$ levels over time, likely reflecting closer follow-up and earlier treatment [6]. Nonetheless, it is of concern that more than $70 \%$ of early metformin users aged $<65$ years without recorded complications or comorbidities did not reach an $\mathrm{HbA}_{1 \mathrm{c}}$ level of $<6.5 \%(<48 \mathrm{mmol} / \mathrm{mol})$ as recommended by treatment guidelines and $25 \%$ still had an $\mathrm{HbA}_{1 \mathrm{c}}$ level of $>7.4 \%$ $(>57 \mathrm{mmol} / \mathrm{mol}$ ) after intensification (upper quartile). One likely explanation is that half the patients in our cohort had $\mathrm{HbA}_{1 \mathrm{c}} \geq 8.0 \%$ ( $\geq 64 \mathrm{mmol} / \mathrm{mol}$ ) (median) and $25 \%$ had $\mathrm{HbA}_{1 \mathrm{c}}$ $\geq 9.2 \%$ ( $77 \mathrm{mmol} / \mathrm{mol}$ ) (upper quartile) at the start of add-on therapy. Alternatively, the number, the dose or the efficacy of the drugs employed prior to intensification may not have fully matched the individual needs of each patient. As the greatest potential for prevention exists early in type 2 diabetes before complications have emerged [4], future observational studies should examine possible reasons why many patients do not attain recommended $\mathrm{HbA}_{1 \mathrm{c}}$ targets following intensification.

Study strengths include a population-based design within the comprehensive Danish public healthcare system and, accordingly, our data reflect actual clinical practice in diabetes care. A limitation was that $\mathrm{HbA}_{1 \mathrm{c}}$ measurements within the right time frame were available only for two-thirds of potentially eligible patients. Moreover, prescription redemption is only a marker of actual drug consumption. Finally, although a wide range of confounding factors were assessed, imperfectly measured, unmeasured (e.g. BMI, beta cell function, lifestyle and socioeconomic measures) or unknown factors still may have affected risk estimates.

In conclusion, the observed glucose-lowering effects in real life are similar for different add-on drug options and comparable with those observed in randomised trials. Still, a substantial proportion of patients with type 2 diabetes in the community setting who receive intensification of metformin monotherapy do not achieve currently recommended $\mathrm{HbA}_{1 \mathrm{c}}$ targets.

Acknowledgements Preliminary data from this study were presented in poster form at the 50th EASD Annual Meeting in Vienna, September 2014.

Funding This study was partly supported by a research grant from Novo Nordisk Scandinavia AB to Aarhus University.

Duality of interest Two of the co-authors (ESB and CLH) are employees of Novo Nordisk Scandinavia AB. As co-authors, they contributed to the design and reporting of the study. By contract, however, the Department of Clinical Epidemiology at Aarhus University had control of the data and retained final authority over design, content and interpretation of the analyses. RWT, LMB, MS, LP, HN and SPJ have reported no personal duality of interest. The Department of Clinical Epidemiology is involved in studies with funding from various companies as research grants to (and administered by) Aarhus University, including the present study. Moreover, the Department of Clinical Epidemiology is a member of the Danish Centre for Strategic Research in Type 2 Diabetes (DD2), supported by the Danish Agency for Science (grant no. 09-067009 and 09-075724). DD2 also is supported by the Danish Health and Medicines Authority, the Danish Diabetes Association and an unrestricted donation from Novo Nordisk A/S. Partners in the DD2 project are listed on the project website at www.DD2.nu. The authors have no non-financial interests that may be relevant to the submitted work.

Contribution statement RWT, LMB, ESB, CLH and SPJ conceived and designed the study. Funding was obtained by RWT, LP, ESB, CLH and SPJ. RWT, LP and SPJ supervised the study. Data was acquired by RWT, LMB, LP and SPJ and was analysed and interpreted by all authors. Statistical analysis was performed by LMB and LP. RWT, ESB, CLH and SPJ drafted the manuscript and LMB, MS, LP and HN critically revised it for important intellectual content. All authors gave final approval of the version to be published. RWT had full access to all the data in the study and takes responsibility for the integrity of the data and the accuracy of the data analysis and is the guarantor.

\section{References}

1. Lipska KJ, Krumholz HM (2014) Comparing diabetes medications: where do we set the bar? JAMA Intern Med 174:317-318

2. Roumie CL, Greevy RA, Grijalva CG et al (2014) Association between intensification of metformin treatment with insulin vs sulfonylureas and cardiovascular events and all-cause mortality among patients with diabetes. JAMA 311:2288-2296

3. Sherifali D, Nerenberg K, Pullenayegum E, Cheng JE, Gerstein $\mathrm{HC}$ (2010) The effect of oral antidiabetic agents on A1C levels: 
a systematic review and meta-analysis. Diabetes Care 33:18591864

4. American Diabetes Association (2015) Standards of medical care in diabetes-2015: summary of revisions. Diabetes Care 38(suppl 1):S4

5. Agency for Healthcare Research and Quality (AHRQ) (2011) Medicines for type 2 diabetes: a review of the research for adults. Available from: http://www.effectivehealthcare.ahrq.gov/search-forguides-reviews-and-reports/?pageaction=displayproduct\&productID $=$ 721, accessed 15 April 2015

6. Thomsen RW, Baggesen LM, Svensson E et al (2015) Early glycaemic control among patients with type 2 diabetes and initial glucose-lowering treatment: a 13-year population-based cohort study. Diabetes Obes Metab 17:771-780

7. Pottegard A, Bjerregaard BK, Larsen MD et al (2014) Use of exenatide and liraglutide in Denmark: a drug utilization study. Eur J Clin Pharmacol 70:205-214

8. Wallia A, Molitch ME (2014) Insulin therapy for type 2 diabetes mellitus. JAMA 311:2315-2325

9. Raebel MA, Ellis JL, Schroeder EB et al (2014) Intensification of antihyperglycemic therapy among patients with incident diabetes: a Surveillance Prevention and Management of Diabetes Mellitus (SUPREME-DM) study. Pharmacoepidemiol Drug Saf 23:699-710 